\title{
Monitoring the role of enantiomers in the surface modification and adsorption process of polymers imprinted by chiral molecules: theory and practice
}

\author{
Marcin Woźnica ${ }^{1}$, Monika Sobiech $^{1, *}$ (D), Norbert Pałka ${ }^{2}$, and Piotr Luliński ${ }^{1}$ \\ ${ }^{1}$ Department of Organic Chemistry, Faculty of Pharmacy, Medical University of Warsaw, Banacha 1, 02-097 Warsaw, Poland \\ ${ }^{2}$ Institute of Optoelectronics, Military University of Technology, Kaliski 2, 00-908 Warsaw, Poland
}

Received: 14 February 2020

Accepted: 5 May 2020

Published online:

14 May 2020

(C) The Author(s) 2020

\begin{abstract}
The objective of this study was to explore the role of enantiomers in the surface modification and adsorption process of polymers imprinted by chiral molecules. Here, $R$-, $S$ - and $R / S$-1-aminoindanes $\left(\mathbf{T}_{R}, \mathbf{T}_{S}\right.$ and $\left.\mathbf{T}_{R / S}\right)$ were used as the model template molecules. Optimization of synthetic protocols facilitated preselection of the most efficient composition for a molecularly imprinted polymer (MIP) methacrylic acid-co-ethylene glycol dimethacrylate-with satisfactory specificity and an imprinting factor equal to 3 . This polymer composition was used further to analyse the effect of configuration of the template on adsorption properties. The results showed preferable adsorption of $R$-enantiomer, $\mathbf{T}_{R}$ in the R-MIP and $S$-enantiomer, $\mathbf{T}_{S}$ in the $S$-MIP but revealed variations in the binding capacities of $\mathbf{T}_{R}$ and $\mathbf{T}_{S}$ in the $R$ - and $S$-cavity (stereoselectivity factor; $k=1.66$ to 1.22 , respectively). The theoretically analysed binding energies $\left(\Delta E_{\mathrm{B}}\right)$ of both $\mathbf{T}_{R}$ and $\mathbf{T}_{S}$ in the $R$-cavity $\left(\Delta E_{\mathrm{B}}=-506.14\right.$ to $\left.-256.77 \mathrm{kcal} \mathrm{mol}^{-1}\right)$ and $S$-cavity $\left(\Delta E_{\mathrm{B}}\right.$ $=-302.42$ to $-347.18 \mathrm{kcal} \mathrm{mol}^{-1}$ ) were in agreement with empirical data. Morphology and porosity analyses revealed the impact of the template molecule on the pore system of the MIP and control (a non-imprinted polymer), but enantiomers were only found to have a negligible role. Binding characterization revealed that the physisorption-governed adsorption process and DubininRadushkevich model fitted the experimental data best. Finally, terahertz spectroscopy was employed to confirm the similarity between both materials imprinted by each enantiomer.
\end{abstract}

Address correspondence to E-mail: monika.sobiech@wum.edu.pl 


\section{Introduction}

Chiral molecules constitute non-overlaid mirror-image forms of enantiomers, which can be identified by rotation of linearly polarized light in the opposite orientation [1]. The physicochemical properties of enantiomers are identical, but their interactions with natural or synthetic chiral materials are diverged [2]. Based on such an observation, the existence of a quantitative structure-activity relationship between the potency of two enantiomers was identified and used to disclose submolecular-level variations in enantiomers [3].

The position of atoms around asymmetric carbon plays a crucial role in decisive consequences when chiral molecules are used to design synthetic materials based on prearranged host-guest complexes such as molecularly imprinted polymers (MIPs). Imprinting technology is a well-established approach that can be used to obtain tailor-made synthetic materials with predetermined properties originating from spatial modification of the polymer's surface by the template [4]. MIPs have attracted attention due to widespread application in biomedical, clinical and toxicological analyses. They have been used as sorbents in separation processes [5-9] and as recognition elements in detection devices [10-12]. They have also been considered as potential drug carriers [13, 14].

One of the crucial challenges in the design of MIPs is comprehensive prediction of the sorption capabilities of resultant polymers [15-17]. In a systematic study of steric and spatial effects of the adsorption process on MIPs [18], Spivak and Campbell pointed out that the most dominant factors contributing to MIP specificity are the molecular volume of the template, the spatial and distal relationship between template substructures and the conformational flexibility. It has also been stated that the most fitting approach for proving the cavity's geometry consists of analysing polymers imprinted by enantiomers, due to identical physicochemical properties, homochiral interactions and the stereogenicity of isomers. These factors have been found to contribute to preferential adsorption for $R$-enantiomer on the $R$ MIP when compared to $S$-enantiomer, and preferential adsorption for $S$-enantiomer on the S-MIP when compared to $R$-one, confirming that spatial requirements govern stereo-differentiation of enantiomers by MIPs [19].
It can also be supposed that the adsorption capacities of $R$ - or $S$-MIPs will be characterized by identical values for $R$-enantiomer on $R$-MIP and $S$-enantiomer on $S$-MIP because surface modification of MIPs by $R$ or $S$-enantiomers should proceed in such a way that mirror-image cavities are formed. However, variability of factors affecting the adsorption process of MIPs could be responsible for the diverse sorption behaviour of enantiomers, revealing different binding capacities of $R$-enantiomer on $R$-MIP when compared to the binding capacities of $S$-enantiomer on $S$ MIP. Ouyang et al. [20] designed thin MIP film sensors for stereo-differentiation of chiral glutamic acid using its $R$ - or $S$-enantiomer as the templates and obtaining $R$-MIP and $S$-MIP, respectively. The adsorption of $R$ - or $S$-enantiomer on $R$-MIP and $S$ MIP was analysed. Electrochemical measurements revealed that the enantiomeric selectivity coefficient of $S$-glutamic acid for $S$-MIP was equal to 24 , but for $R$-glutamic acid on $R$-MIP, it only equalled 15 . It was concluded that the higher enantiomeric selectivity coefficient of S-MIP might be assigned to the difference in film thickness because of the enantiomorphic property of $S$-glutamic acid, which produced thicker film. Sekine et al. [21] investigated the chiral discriminative gate effects of MIPs prepared on indium tin oxide using $R$ - or $S$-phenylalanine anilide as the templates, respectively. Cyclic voltammetry was employed for analysis, and the results (expressed as the relative change in maximum anodic current in the presence of $R$ - or $S$-phenylalanine anilide) revealed significant differences between adsorption of $S$ enantiomer on S-MIP (-35.3\%) when compared to $R$-enantiomer on $R$-MIP (-27.9\%). It was concluded that the current was influenced by the thickness and density of MIP on indium tin oxide. Thus, it was stated that enantiomers affected the morphology of material, which was, in turn, responsible for differences in the adsorption of $R$ - or $S$-enantiomers. Finally, Terracina et al. [22] found differences in theoretically calculated binding energy values between tyrosine derivatives and the cavities imprinted by respective tyrosine $R$ - or $S$-enantiomers. These variations resulted from different positioning of monomer residues in the polymer net. It was underlined that both cavities were not perfect mirror images of each other, and disparities in the microenvironment of both cavities extended beyond chirality. It was suggested that the degree of randomness of the starting orientation of the system was 
responsible for producing different final geometries. Similar observations were found by Sobiech et al. [23]. Computationally constructed models of cavities imprinted by $R$ - or S-2-amino-1-phenylethanol templates were characterized by different binding energies of $R$ - or $S$-octopamine, respectively. Nevertheless, this observation was not investigated further [24]. The above-mentioned examples, although scarce in the literature, indicate that the adsorption process of enantiomers on polymers imprinted by a chiral template is more complex than was first thought and that factors related to the morphology and structure of resultant polymers should be comprehensively monitored.

This study focuses on monitoring the role of enantiomers in surface modification of resultant polymers during the imprinting process as well as during the adsorption process, on polymers imprinted by a chiral model template molecule. For this purpose, simple models of a low-molecular-weight biomolecule of $R-, S$ - and $R / S$-1-aminoindane (coded below as $\mathbf{T}_{R}, \mathbf{T}_{S}$ and $\mathbf{T}_{R / S}$, respectively) were employed as the templates because of the limited number of interactions with monomers [in the prepolymerization complex (PC)] or with the monomer residues (in the polymer matrix during the adsorption process), as well as due to the conformational stability of the compound. The aim was realized in two main steps. The first step was devoted to theoretical and experimental analysis of interactions in the PCs, in order to identify submolecular factors responsible for forming the stereospecific surface of polymers. The second step comprised theoretical analysis of the binding energies of enantiomers in the imprinted adsorption site models and experimental determination of the binding capacities of enantiomers on the respective imprinted materials, to ascertain the relationship to their morphology and structure. Here, in addition to other techniques commonly used to characterize MIPs, terahertz radiation $(0.1-3 \mathrm{THz})$ was applied to confirm the structural similarity of imprinted materials. Terahertz spectroscopy is widely used to study the internal structure of many materials, including polymers [25], which are highly transparent in this spectral range, but to the best of our knowledge, this spectroscopic technique has never been used for analysis of MIPs. The noteworthy approach presented in this paper provides comprehensive knowledge relating to surface modification of polymers imprinted by the chiral template, and it explains fundaments of the adsorption process in a model system.

\section{Experimental section}

\section{Materials}

The $R / S$-1-aminoindane $\left(\mathbf{T}_{R / S}\right), R$-1-aminoindane $\left(\mathbf{T}_{R}\right)$ and $S$-1-aminoindane $\left(\mathbf{T}_{S}\right)$ templates were obtained from Alfa Aesar (Karlsruhe, Germany). The functional monomers [allylamine (1), itaconic acid (2) and methacrylic acid (5)] came from Sigma-Aldrich (Steinheim, Germany). The acrylic acid (3) and 4-vinylbenzoic acid (4) were obtained from Alfa Aesar (Karlsruhe, Germany). The cross-linker (ethylene glycol dimethacrylate; EGDMA) was purchased from Sigma-Aldrich (Steinheim, Germany). The solvents [methanol, acetone and formic acid (96\%)] were purchased from POCh (Gliwice, Poland). The polymerization reaction initiator (2,2-azobisisobutyronitrile) came from Merck (Darmstadt, Germany). The HPLC, MS-grade methanol and acetonitrile were obtained from Sigma-Aldrich (Steinheim, Germany). Ultra-pure water, delivered from a Hydrolab HLP 5 system (Straszyn, Poland), was used to prepare the water solutions. Preparation of the stock solutions is described in the Supplementary Information section (S1).

\section{Synthesis of polymers}

The MIPs (coded as $\mathrm{MIP}_{R / \mathcal{S}}-\mathrm{MIP}_{\boldsymbol{R} / \mathcal{S}}, \mathrm{MIP}_{\boldsymbol{R}}$ and $\mathrm{MIP5}_{S}$ ) and corresponding non-imprinted polymers (NIPs) (NIP1-NIP5) were prepared according to the synthetic protocols described in the Supplementary Information section (S2). Reference NIPs were prepared under the same polymerization conditions but without the template molecule and were treated in the same way as the corresponding MIPs. The amounts of reagent used for preparation of the different types of polymers are listed in Table S1. Two synthetic series were prepared for all the polymers.

\section{Instruments}

UV-Vis spectroscopy was used for binding studies of $\mathrm{MIP1}_{R / S}-\mathrm{MIP} 5_{R / S}$ and NIP1-NIP5 during preselection of the polymer composition. The analysis was performed on a UV-2600 spectrophotometer (Shimadzu, 
Duisburg, Germany). Details of analytical measurements are described in the Supplementary Information section (S2).

An ultra-fast liquid chromatography (UFLC) system, coupled with an LCMS-2020 mass spectrometry detector (Shimadzu, Duisburg, Germany), was used for analysis of $\mathbf{T}_{R}$ and $\mathrm{T}_{S}$ on MIPs and NIPs. The system consisted of an LC 20AD pump, DGU-20A SR degassing unit, CTO 10AS VP oven, SPD-20A UV-Vis detector and SIL 20AC HT autosampler (Shimadzu, Duisburg, Germany). Chromatographic separation was performed using a Kinetex ${ }^{\circledR}$ F5 stainless-steel column $(50 \mathrm{~mm} \times 2.1 \mathrm{~mm}$ ID and $2.6 \mu \mathrm{m}$; Phenomenex, Torrance, CA, USA), preceded by a SecurityGuard $^{\circledR}$ ULTRA column protector (Phenomenex, Torrance, CA, USA). The LCMS system operating conditions were as follows: nebulizer gas: nitrogen $\left(1.5 \mathrm{~L} \mathrm{~min}^{-1}\right)$; drying gas: nitrogen $\left(15 \mathrm{~L} \mathrm{~min}^{-1}\right)$; interface temperature: $350{ }^{\circ} \mathrm{C}$; DL temperature: $250{ }^{\circ} \mathrm{C}$; heat block temperature: $200{ }^{\circ} \mathrm{C}$; detector voltage: $1.2 \mathrm{kV}$; interface voltage: $4.5 \mathrm{kV}$. The LCMS data were collected and interpreted with LabSolution software.

For the purpose of mass spectrometry determination of $\mathbf{T}_{R}$ and $\mathbf{T}_{S}$, the five-point calibration lines were constructed as a function of intensity $(y)$ versus concentration $(x)$. The range of calibration lines, equation, linearity $\left(r^{2}\right)$, limit of quantification and limit of detection (in $\mu \mathrm{mol} \mathrm{L}^{-1}$ ) were as follows: $\mathbf{T}_{R}$ : $0.1-10 \mu \mathrm{mol} \mathrm{L}^{-1}, y=146199 x-2321,0.997,0.412$, 0.136; $\mathrm{T}_{S}: 0.1-10 \mu \mathrm{mol} \mathrm{L} \mathrm{L}^{-1}, y=151000 x+5319$, $0.995,0.587,0.194$. The mobile phase consisted of an acetonitrile-water system $(55: 45 \mathrm{v} / \mathrm{v})$, delivered at a flow rate of $0.2 \mathrm{~mL} \mathrm{~min}{ }^{-1}$ at $40{ }^{\circ} \mathrm{C}$. The injection volume was $5 \mu \mathrm{L}$. The run-time was $5 \mathrm{~min}$. Mass spectrometry was performed during positive ion detection: $[\mathrm{M}+\mathrm{H}]^{+}$. The following ions for 1-aminoindane were monitored: $\mathrm{m} / \mathrm{z} 117$ and $\mathrm{m} / \mathrm{z} 134$.

Scanning electron microscopy (SEM) analyses were performed in the Faculty of Chemistry at the University of Warsaw, Poland. The surfaces of imprinted and non-imprinted materials were studied using a Merlin FE-SEM (Zeiss, Oberkochen, Germany). The samples were $\mathrm{Au} / \mathrm{Pd}$ sputter-coated before analysis. Specific surface area, pore volume and pore size were determined using the $\mathrm{N}_{2}$ adsorption isotherm at $77 \mathrm{~K}$ on the ASAP 2420 (Micromeritics Inc., Norcross, GA, USA) in the Faculty of Chemistry at Maria Curie-Skłodowska University, Lublin, Poland. The ${ }^{1} \mathrm{H}$ NMR spectra were recorded with a Bruker Avance DMX 400 spectrometer (Bruker, Mannheim, Germany) in the Faculty of Pharmacy at the Medical University of Warsaw. The $\delta$ ppm chemical shifts were referenced to tetramethylsilane. Terahertz spectroscopy was performed at the Military University of Technology Institute of Optoelectronics, Warsaw, Poland. A TDS-based spectrometer was used for terahertz measurements (Teraview TPS Spectra 300, Cambridge, UK) in transmission. Samples were prepared as $400 \mathrm{mg}$ pellets and pressed from a mixture of $80 \%$ polyethylene and $20 \%$ examined polymer material. To observe repeatability, two samples of each kind were prepared. The reference pellet was a 320-mg sample manufactured from pure polyethylene.

\section{Binding studies}

Stationary binding experiments were performed to evaluate the binding ability of MIP1 $1_{R / S}-M I P 5_{R / S}$ and NIP1-NIP5 towards $\mathrm{T}_{R / S}$, and for MIP5 $5_{R}, \mathrm{MIP}_{S}$ and NIP5 towards $\mathbf{T}_{R}$ and $\mathrm{T}_{S}$. Polypropylene tubes (10$\mathrm{mL}$ volume) were filled with $10 \mathrm{mg}$ of MIP or NIP particles. To each tube, a 5-mL volume of methanolwater $(85: 15 \mathrm{v} / \mathrm{v})$ standard solution of $\mathrm{T}_{R / S}$ $\left.(10 \mu \mathrm{mol} \mathrm{L})^{-1}\right), \mathbf{T}_{R}$ or $\mathbf{T}_{S}\left(10\right.$ or $\left.2 \mu \mathrm{mol} \mathrm{L}^{-1}\right)$ was added. The tubes were sealed and oscillated by a shaker at room temperature for $3 \mathrm{~h}$ prior to centrifugation $(6000 \mathrm{rpm} ; 20 \mathrm{~min})$. Aliquots of the supernatant $(0.7 \mathrm{~mL}$ or $5 \mu \mathrm{L})$ were used to analyse unbound amounts of each compound via UV-Vis spectroscopy (for $\mathrm{MIP}_{R / S}-\mathrm{MIP}_{R / S}$ and NIP1-NIP5) or UFLC-MS (for MIP5 $5_{R}, \mathrm{MIP}_{S}$ and NIP5) using the calibration lines. The amounts of analyte bound to the polymer were calculated by subtracting the unbound amount from the initial amount. All measurements were made in triplicate for two synthetic series of all polymers.

Parameters characterizing the polymers [e.g. binding capacities $(B)$, distribution coefficients $\left(K_{D}\right)$ and imprinting factors (IFs)] were computed on the basis of the binding measurements, as described in the Supplementary Information section (S3). For the isotherm evaluation, the same protocol as the above was conducted, but to each tube, a $5-\mathrm{mL}$ volume of methanol-water $(85: 15 \mathrm{v} / \mathrm{v})$ standard solution of $\mathbf{T}_{R}$ (conc. of $0.25,0.5,0.75,1,1.25,2.5,5,10 \mu \mathrm{mol} \mathrm{L}{ }^{-1}$ ) was added. The models used to fit the adsorption data were expressed by equations defined in the Supplementary Information sections (S3) and (S4). 


\section{Molecular modelling}

The results of the molecular modelling reported in this study were obtained according to data described in the Supplementary Information section (S5). In order to analyse the adsorption properties at a molecular level, models of PCs and enantiomeric binding sites were created in two main steps for $\mathrm{MIP}_{R}$ and $\mathrm{MIP} 5_{S}$, formed by imprinting of $\mathbf{T}_{R}$ or $\mathbf{T}_{S}$ in the prepolymerization mixture of monomer (5) and EGDMA.

Models for the PCs were set up first. To obtain starting structures for the PCs, boxes were created with the template molecule $\left(\mathbf{T}_{R}\right.$ or $\left.\mathbf{T}_{S}\right)$ surrounded by ten monomer molecules (5) and 102 molecules of solvent (methanol). The number of methanol molecules was chosen to mimic molar ratios used during the synthetic process. Monomers and solvent molecules were located randomly around the template, and intermolecular interactions were obtained during the energy minimization procedure. Next, four molecules of (5) were selected from each minimized system, taking into account the molar ratio used in the synthetic procedure and the strongest interactions with the template. Twenty molecules of cross-linker (EGDMA) were then added, reflecting the stoichiometry of the synthetic procedure (to create the polymerization system formed around the template), and minimization of the energy was performed. Molecular dynamics (MD) simulation of the system was subsequently carried out. For PCs created in this way, the optimization procedure was repeated.

Binding cavities in $\mathrm{MIP}_{R}$ and $\mathrm{MIP}_{S}$ were constructed on the basis of the average structures of the systems. Double bonds in the monomer and crosslinker molecules were replaced by single bonds. The hydrogenation process that approximates formation of single $\mathrm{C}-\mathrm{C}$ bonds during polymerization was carried out by adding hydrogen atoms to unsaturated bonds of vinyl groups of monomers [23, 26-28]. The template was removed from the cavities, and the empty spaces were proposed as the computer models of binding sites in the polymer matrices. Simulation of the $\mathbf{T}_{R}$ and $\mathbf{T}_{S}$ adsorption process was then performed. The $\mathbf{T}_{R}$ or $\mathbf{T}_{S}$ molecules used as templates during formation of the polymer cavity were inserted into the models of $\mathrm{MIP}_{R}$ or MIP5 $5_{S}$ empty cavities. The solvent (consisting of 418 molecules of methanol and 167 molecules of water) was added to form boxes, and MD simulations were carried out (mimicking the experimental conditions of adsorption). During computations, constraints were set on the monomers and cross-linker molecules using harmonic distance restraints (with a force constant of $100 \mathrm{kcal} \mathrm{mol}^{-1} \AA^{2}$ ) in order to immobilize the 3D structure of MIP cavities and to determine the binding energies $\left(\Delta E_{\mathrm{B}}\right)$, as described in the Supplementary Information section (eq. S4). Radial distribution function (RDF) analysis was performed using BIOVIA Discovery Studio software [29].

\section{Results and discussion}

\section{Composition of the polymer matrix}

Synthesis of efficient MIPs using a non-covalent approach required careful optimization of the prearranged system between the template and the monomer. Thus, the prepolymerization system was firstly optimized to preselect the monomer which could form the most stable interactions with the template and, as a result, provide the most efficient MIP. For this purpose, the racemic template $\left(T_{R / S}\right)$ was selected, and five different monomers were tested [basic: allylamine (1), acidic: itaconic acid (2), acrylic acid (3), 4-vinylbenzoic acid (4) and methacrylic acid (5)]. Synthesis was carried out as a thermal, radical process in the presence of EGDMA acting as the crosslinker, in order to form bulk polymers and obtain materials coded as MIP1 $1_{R / S}-\mathrm{MIP} 5_{R / S}$ and NIP1-NIP5.

Stationary binding experiments were carried out to determine the binding ability of $M I P 1_{R / S}-M I P 5_{R / S}$ and NIP1-NIP5 towards $T_{R / s}$. The binding capacities $\left(B ; \mu \mathrm{mol} \mathrm{g}{ }^{-1}\right)$ of MIPs and NIPs were calculated according to eq. S1; distribution ratios $\left(K_{D} ; \mathrm{L} \mathrm{g}^{-1}\right)$ were calculated according to eq. S2; and imprinting factors (IFs) were calculated according to eq. S3. Table 1 presents the $B$ of MIP1 $1_{R / S}-\mathrm{MIP}_{R / S}$ and NIP1NIP5, the $K_{D}$ and the IF towards $\mathbf{T}_{R / S}$.

As can be seen, the physicochemical nature of monomers plays a crucial role in the binding ability of resultant MIPs. The basic monomer (1) built into the structure of $M I P 1_{R / S}$ revealed the lowest $B$ of $\mathbf{T}_{R / S}$. This fact could be explained by the weak interactions between $T_{R / S}$ and monomer (1) during formation of the PC, as well as during the adsorption process. Significantly, higher $B$ values were noted for all MIPs synthesized from acidic monomers (2)-(5). It could be supposed that the strong interactions between the 
Table 1 Binding capacities, $B$ of $R / S$-1-aminoindane, $\mathbf{T}_{R / S}$ (template) on MIP1 $1_{R / S^{-}}$ $\mathrm{MIP}_{R / S}$ and NIP1-NIP5, distribution ratios, $K_{D}$, and imprinting factors, IFs (conc. $10 \mu \mathrm{mol} \mathrm{L}{ }^{-1}, n=3$ )

\begin{tabular}{|c|c|c|c|c|c|}
\hline \multirow[t]{2}{*}{$\begin{array}{l}\text { No. of polymers } \\
\text { (monomer used during synthesis) }\end{array}$} & \multicolumn{2}{|c|}{$\begin{array}{l}\text { Binding capacities } \pm \text { S.D. } \\
\left(B, \mu \mathrm{mol} \mathrm{g}^{-1}\right)\end{array}$} & \multicolumn{2}{|c|}{$\begin{array}{l}\text { Distribution ratio } \\
\left(K_{D}, \mathrm{~L} \mathrm{~g}^{-1}\right)\end{array}$} & \multirow[t]{2}{*}{ IF } \\
\hline & $\mathrm{MIP}_{R / S}$ & NIP & $\mathrm{MIP}_{R / S}$ & NIP & \\
\hline 1 Allylamine & $0.580 \pm 0.010$ & $1.051 \pm 0.058$ & 0.090 & 0.190 & 0.47 \\
\hline 2 Itaconic acid & $3.70 \pm 0.27$ & $3.73 \pm 0.77$ & 1.738 & 1.814 & 0.96 \\
\hline 3 Acrylic acid & $2.16 \pm 0.32$ & $2.398 \pm 0.059$ & 0.415 & 0.507 & 0.82 \\
\hline 4 4-Vinylbenzoic acid & $3.82 \pm 0.60$ & $2.89 \pm 0.12$ & 2.041 & 0.774 & 2.64 \\
\hline 5 Methacrylic acid & $2.19 \pm 0.19$ & $0.980 \pm 0.023$ & 0.310 & 0.103 & 3.00 \\
\hline
\end{tabular}

$-\mathrm{NH}_{2}$ group from $\mathrm{T}_{R / S}$ and $-\mathrm{COOH}$ group from the monomer stabilized the PC and, consequently, that the strong interactions between the $-\mathrm{NH}_{2}$ group from $\mathbf{T}_{R / S}$ and acidic residues in the polymer network governed the adsorption process. A similar trend was observed for all NIPs except NIP5, revealing more complex non-specific adsorption on polymers prepared from monomers (1)-(4).

It should be underlined that adsorption on NIPs could be considered as non-specific. The structure of monomer residues in the polymeric network and the impact of monomers on the pore system of resultant polymers played a crucial role in the adsorption process. The presence of two carboxylic groups from (2) could be responsible for the highest value of nonspecific adsorption on NIP2. However, the specificity of MIP2 was very low, confirming previous findings [30]. The presence of an aromatic ring from (4) could have affected the adsorption process, explaining acceptable specificity of MIP4. Although scarce in the literature, MIPs possessing 4-vinylbenzoic acid residues have been found to be characterized by sufficient specificity [31]. In this study, the lowest values of $B$ were noted for NIP3 and NIP5 prepared from monomers (3) or (5), respectively, but only MIP5 revealed satisfactory specificity. Here, polymerization in the presence of (3) or (5) affected the pore system formed in the bulk polymer, providing materials characterized by different binding properties [32]. NIP5 showed the lowest $B$. This fact was responsible for the high specificity of $M I P 5_{R / S}$ with an IF equal to 3 . Thus, the polymer synthesized from monomer (5) was characterized by the highest specificity towards the template and was selected as the most appropriate matrix for the purpose of further studies.

\section{Theoretical analysis of the prearrangement} of monomers around the chiral template

To provide an insight into the impact of the absolute configuration of the $\mathbf{T}$ on recognition properties of MIPs, we scrutinized intermolecular interactions between $\mathbf{T}_{R}$ or $\mathbf{T}_{S}$ and PC components, viz. monomer (5) and cross-linker EGDMA. The complexes were coded as $P C 5_{R}$ and $\mathrm{PC}_{S}$, respectively, for resultant $\mathrm{MIP}_{R}$ or $\mathrm{MIP5}_{s}$. These interactions are shown in Fig. 1a, b. Complexation energies $\left(\Delta E_{C}\right)$ computed according to eq. $S 4$ were analysed for $T_{R}$ and $T_{S}$. A lower $\Delta E_{C}$ value was obtained for the $\mathrm{T}_{R}$ complex ( $-1853.06 \mathrm{kcal} \mathrm{mol}^{-1}$ ) compared to the $\mathrm{T}_{S}$ one $\left(-1697.11 \mathrm{kcal} \mathrm{mol}^{-1}\right)$. In $\mathrm{PC}_{\boldsymbol{R}}$ (the last conformation after MD simulation), three classical hydrogen bonds were formed between $\mathrm{N}$ and $\mathrm{H}$ atoms from the $-\mathrm{NH}_{2}$ group of $\mathrm{T}_{R}$, and between $\mathrm{O}$ and $\mathrm{H}$ atoms from the $-\mathrm{COOH}$ groups from two monomer molecules (2.11, 2.18 and $2.30 \AA$, respectively). Additionally, $\mathbf{T}_{R}$ interacted with EGDMA. Two hydrophobic interactions between $\pi$-electrons and the $-\mathrm{CH}_{3}$ group of EGDMA were observed (4.67 and $5.18 \AA$ ). In the $\mathrm{PC}_{S}$ complex (the last conformation after MD simulation), the $\mathrm{N}$ atom of the $-\mathrm{NH}_{2}$ group from $\mathbf{T}_{S}$ formed one classical hydrogen bond with the $-\mathrm{COOH}$ group from the monomer $(2.30 \AA)$, and the acyclic ring of $\mathbf{T}_{S}$ created a hydrophobic interaction with the $-\mathrm{CH}_{3}$ monomer group (5.47 $\AA$ ). $\Delta E_{C}$ values and interactions observed between the $\mathrm{T}$ and $\mathrm{PC}$ components showed that $\mathrm{PC}_{R}$ was more stable than $P C 5_{s}$. It was also observed that the way monomers were arranged around the $\mathrm{T}$ was different in $\mathrm{PC} 5_{R}$ and $\mathrm{PC}_{S}$ complexes.

Additionally, average spatial distributions of functional monomers around the $\mathbf{T}$ were investigated by RDFs in order to confirm the nature of templatemonomer complexes. The RDF values confirmed stronger interactions between $\mathbf{T}_{R}$ and monomer (5) 
(a)

(b)

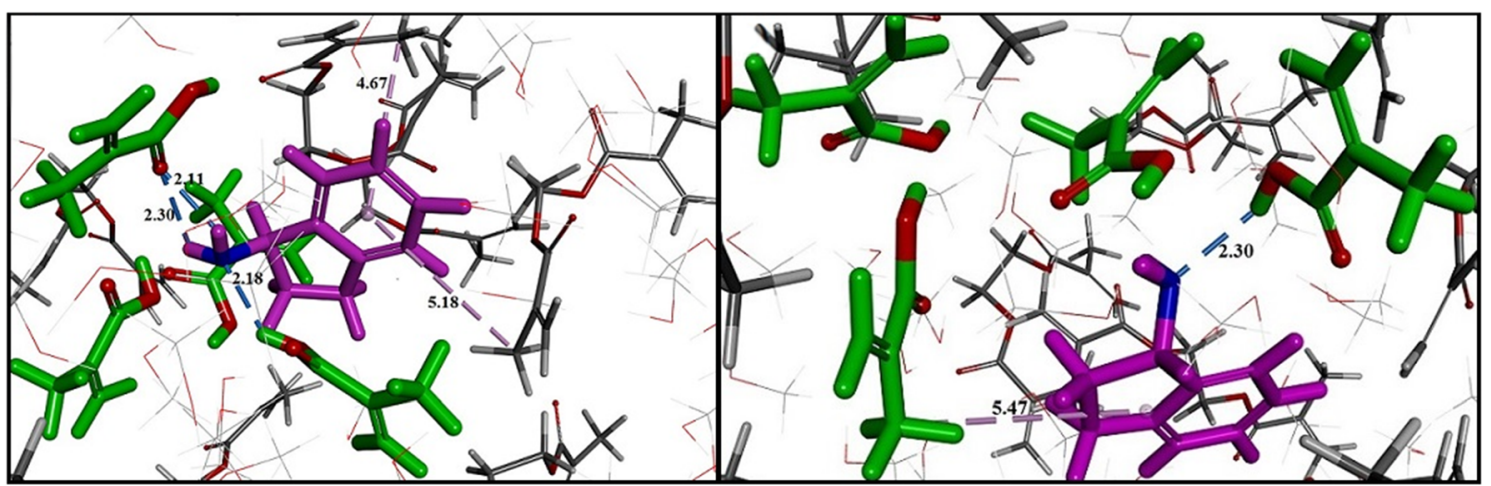

Figure 1 Prepolymerization complexes of $\mathbf{a} \mathrm{PC}_{\boldsymbol{R}}$ and $\mathbf{b} \mathrm{PC}_{S}$. The template is shown as purple and monomers as green molecules. The classical hydrogen bonds are shown as the blue than between $\mathbf{T}_{S}$ and monomer (5) (Fig. 2). Higher RDF values were observed for $\mathbf{T}_{R}$ (Fig. 2a). The types of hydrogen bonds observed in $\mathrm{PC}_{R}$ and $\mathrm{PC}_{S}$ were also confirmed by RDF analysis (Fig. 2a, b).

\section{Experimental examination of interactions between monomers and the chiral template}

${ }^{1} \mathrm{H}$ NMR spectroscopy is considered to be a versatile tool for analysis of the interactions between PC components [33]. Chemical proton shifts indicate extended interaction between interacting molecules and provide an insight into the impact of PC stability on the imprinting effect. Here, ${ }^{1} \mathrm{H}$ NMR spectroscopy was employed to analyse the molecular regions responsible for PC stabilization. Chemical shifts were observed in a titration of $\mathbf{T}_{R}$ or $\mathbf{T}_{S}$ enantiomers with monomer (5), forming subsequent complexes of $\mathrm{PC}_{R}$ and $\mathrm{PC}_{s}$. The ${ }^{1} \mathrm{H}$ NMR spectra of $\mathrm{PC}_{R}$ and $\mathrm{PC}_{S}$ were recorded and compared to those obtained for neat $\mathbf{T}_{R}$ (Fig. 3a) and $\mathbf{T}_{S}$ (Fig. 3b) and monomer (5).

As can be seen, formation of $\mathrm{PC} 5_{R}$ or $\mathrm{PC} 5_{S}$ affected proton signals from the $\mathrm{T}$. The largest shift was observed for $\mathrm{H} 8$ protons from the $-\mathrm{NH}_{2}$ group of the $\mathrm{T}$, which interacted with the $\mathrm{H}^{\prime}$, proton from monomer (5). This indicated that strong electrostatic interactions between $-\mathrm{COOH}$ and $-\mathrm{NH}_{2}$ were responsible for stabilizing PCs. Shifts of $\mathrm{H} 7$ and both $\mathrm{H} 6$ protons from the acyclic ring of the $\mathbf{T}$ were also detected. A negligible shift of protons in the aromatic system was observed, but the $\mathrm{H} 5$ proton in the acyclic ring was unaffected. The vinyl protons of $\mathrm{H}^{\prime}$ from monomer (5) were significantly shifted into the lower values of $\delta$. This observation suggested that in the dashed lines, and hydrophobic interactions are shown as the pink dashed lines. Their lengths are given in $\AA$.

prepolymerization mixture, the $\mathbf{T}$ interacted with monomer mainly through the $-\mathrm{NH}_{2}$ and $-\mathrm{COOH}$ groups, respectively, affecting nearby protons. The same observation has been described by Long et al. [30].

Next, a comparison was made between the results of the theoretical analysis of interactions in the PC and the experimental results of ${ }^{1} \mathrm{H}$ NMR analysis. Both sets of results correlated well, and both confirmed that classical hydrogen bonds between $-\mathrm{NH}_{2}$ from the $\mathrm{T}$ and $-\mathrm{COOH}$ from monomer (5) were responsible for PC stabilization.

Finally, the ${ }^{1} \mathrm{H}$ NMR spectra of $\mathrm{PC}_{R}$ and $\mathrm{PC}_{S}$ were compared. Chemical shifts in protons substituted in the acyclic ring of the T (H7 and H6) were similar in both PCs. Subtle differences were noted in the chemical shifts of signals derived from $\mathrm{H} 3^{\prime} / \mathrm{H} 8$ in the PC stoichiometry equal to 1:1 (8.47 and $8.65 \mathrm{ppm}$ for $\mathrm{PC} 5_{R}$ and $P C 5_{S}$, respectively) (see arrows in Fig. 3) and 1:2 (9.40 and $9.69 \mathrm{ppm}$ for $\mathrm{PC} 5_{R}$ and $\mathrm{PC} 5_{S}$, respectively). Shifting of the $\mathrm{H} 8$ proton of $\mathrm{T}_{R}$ went from $2.13 \mathrm{ppm}$ to $8.47 \mathrm{ppm}$ in $\mathrm{PC}_{R}$ (equimolar stoichiometry), and that of $\mathbf{T}_{S}$ went from $2.01 \mathrm{ppm}$ to $8.65 \mathrm{ppm}$ in $\mathrm{PC}_{S}$ (equimolar stoichiometry). This observation is difficult to explain, and its relation to the strength of interactions in PCs can only be speculated upon.

\section{Effect of absolute template configuration on the binding property of polymers}

Next, the impact of the absolute configuration of the $\mathbf{T}$ on binding capacities of resultant polymers was investigated. Here, the prepolymerization system of 

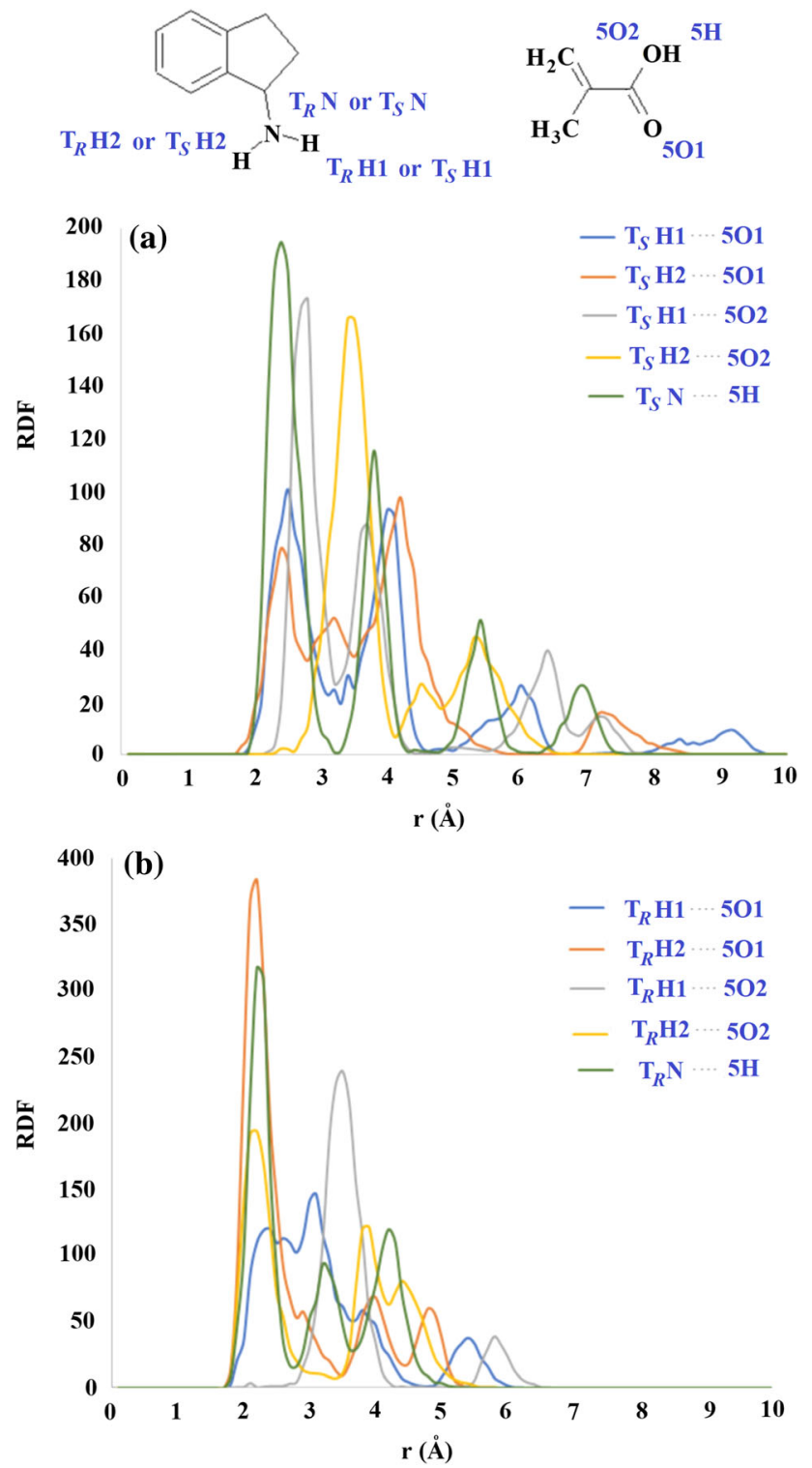

Figure 2 Atoms of $\mathbf{T}_{R}$ or $\mathbf{T}_{S}$ and (5) used during RDF analysis (at the top), a RDFs for the nitrogen and hydrogen atoms $\left(\mathrm{T}_{R} \mathrm{~N}\right.$, $\left.\mathbf{T}_{R} \mathrm{H} 1, \mathbf{T}_{R} \mathrm{H} 2\right)$ of $\mathbf{T}_{R}$ to oxygen and hydrogen atoms (5O1, 5O2, $5 \mathrm{H})$ of (5), b RDFs for the nitrogen and hydrogen atoms $\left(\mathrm{T}_{S} \mathrm{~N}\right.$, $\left.\mathbf{T}_{S} \mathrm{H} 1, \mathbf{T}_{S} \mathrm{H} 2\right)$ of $\mathbf{T}_{S}$ to oxygen and hydrogen atoms $(5 \mathrm{O} 1,5 \mathrm{O} 2$, $5 \mathrm{H})$ of (5).

monomer (5) was taken into account. During formation of PCs, enantiomers were employed independently as templates, viz. $\mathbf{T}_{R}$ or $\mathbf{T}_{S}$, and after polymerization, two independent polymers $\left(\mathrm{MIP}_{\boldsymbol{R}}\right.$ and $\mathrm{MIP}_{S}$ ) were obtained together with a NIP. Stationary binding experiments for $\mathrm{MIP}_{R}, \mathrm{MIP}_{S}$ and NIP5 towards $\mathbf{T}_{R}$ and $\mathbf{T}_{S}$ were then carried out. In order to emphasize specificity of the imprinted materials (which is higher in lower concentration ranges of analytes), the concentration of $\mathbf{T}_{R}$ or $\mathbf{T}_{S}$ standard solution was as low as $2 \mu \mathrm{mol} \mathrm{L}{ }^{-1}$. The $B$ of MIPs and NIPs was calculated according to eq. S1; $K_{D}$ was calculated according to eq. S2; and IFs were calculated according to eq. S3. Table 2 presents the following: $B$ of MIP5 $5_{R}, \mathrm{MIP}_{S}$ and NIP5; $K_{D} ;$ IFs; and stereoselectivity coefficients ( $k$ towards $\mathbf{T}_{R}$ and $\mathbf{T}_{S}$ ).

As can be seen, MIPs presented higher $B$ than NIPs. This means that the imprinting process was carried out successfully even when each particular enantiomer was used as the template. The specificity of respective MIPs for adsorption of the same enantiomer used as the template was high, with the following values: IF for $\mathrm{MIP}_{R}$ : 3.96 ; IF for $\mathrm{MIP}_{s}: 3.23$. Enantiomeric differentiation (expressed by the enantioselectivity coefficient $(k)$, defined for MIP5 $5_{R}$ as the ratio of the $K_{D}$ of $\mathbf{T}_{R}$ to $\mathbf{T}_{S}$, and for $M I P 5_{S}$ as the ratio of the $K_{D}$ of $\mathbf{T}_{S}$ to $\mathbf{T}_{R}$ ) of each MIP was also clearly observed. This was confirmed by higher $K_{D}$ for $\mathbf{T}_{\boldsymbol{R}}$ and lower $K_{D}$ for $\mathbf{T}_{S}$ on MIP5 $5_{R}$ (with $k=1.66$ ), as well as higher $K_{D}$ for $\mathbf{T}_{S}$ and lower $K_{D}$ for $\mathbf{T}_{R}$ on $M I P 5_{S}$, (with $k=1.22$ ). Spivak and Campbell [18] analysed binding behaviour just using a model of an S-1aminoindane imprinted polymer. The polymer was evaluated as a stationary phase in the chromatographic column, resulting in a similar value of enantiomeric differentiation (equal to 1.44). However, no $R$-1-aminoindane imprinted polymer was synthesized, so the researchers omitted to analyse factors responsible for diverse sorption behaviour of $R$ enantiomer on $R$-MIP when compared to $S$-enantiomer on S-MIP.

Next, characterization of the adsorption process of $\mathrm{T}_{R}$ on $\mathrm{MIP5}_{R}$ and NIP5 was provided. This system was selected due to the highest value of IF. The $B$ was analysed in a low range of concentrations of $\mathrm{T}_{R}$ between 0.25 and $10 \mu \mathrm{mol} \mathrm{L}^{-1}$. The obtained data were fitted into two models, namely those of Langmuir and Dubinin-Radushkevich. The Langmuir model was employed first [34]. This model is based on the concept that solid surfaces possess finite adsorption sites. The surface did not adsorb solute when all the adsorption sites were filled. It was also assumed that adsorption sites were homogeneous and that the monolayer was formed (excluding interactions between adsorption sites). The linear form of Langmuir isotherm is presented in eq. S5. The plot of $1 / B$ versus $1 / C_{f}$ gave a straight line with a slope and intercept of $1 / Q_{\max }$ and $1 / Q_{\max } \cdot K_{\mathrm{L}}$, respectively. The Langmuir constant $\left(K_{\mathrm{L}}\right)$ related to 
(a)

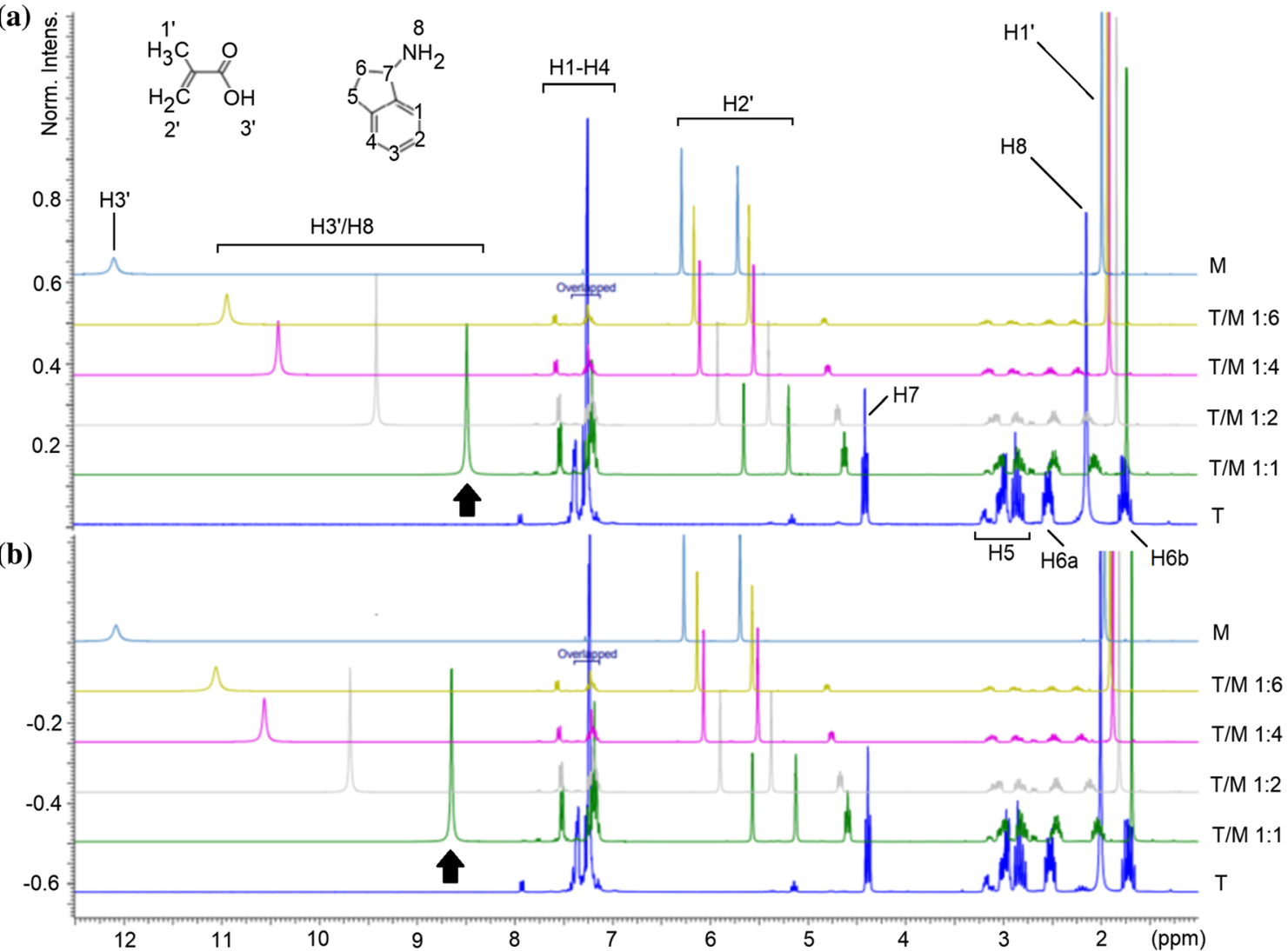

Figure $3{ }^{1} \mathrm{H}$ NMR spectra of complexes between 1-aminoindane (T): $\mathbf{T}_{R}(\mathbf{a})$ or $\mathbf{T}_{S}(\mathbf{b})$, and methacrylic acid (M).

Table 2 Binding capacities, $B$ of $R$-1-aminoindane, $\mathbf{T}_{R}$, and $S-1$ aminoindane, $\mathbf{T}_{S}$ on $\mathrm{MIP}_{R_{R}}$ MIP5 $5_{S}$ and NIP5, distribution ratios, $K_{D}$, imprinting factors, IFs (conc. $2 \mu \mathrm{mol} \mathrm{L}{ }^{-1}, n=3$ ) and adsorption data (conc. $0.25-10 \mu \mathrm{mol} \mathrm{L}{ }^{-1}, n=3$ ) fitted into mathematical models for $\mathrm{T}_{R}$ on $\mathrm{MIP}_{R}$ and NIP5

\begin{tabular}{|c|c|c|c|c|c|c|c|c|}
\hline \multirow[t]{2}{*}{ Solution } & \multicolumn{3}{|c|}{$\begin{array}{l}\text { Binding capacities } \pm \text { S.D. } \\
\left(B, \mu \mathrm{mol} \mathrm{g}^{-1}\right)\end{array}$} & \multicolumn{3}{|c|}{$\begin{array}{l}\text { Distribution ratio } \\
\left(K_{D}, \mathrm{~L} \mathrm{~g}^{-1}\right)\end{array}$} & \multicolumn{2}{|l|}{ IF } \\
\hline & $\mathrm{MIP}_{R}$ & $\mathrm{MIP}_{S}$ & NIP5 & $\mathrm{MIP5}_{R}$ & $\mathrm{MIP}_{S}$ & NIP5 & $\mathrm{MIP}_{R}$ & $\mathrm{MIP}_{s}$ \\
\hline $\mathbf{T}_{R}$ & $0.170 \pm 0.007$ & $0.134 \pm 0.002$ & $0.068 \pm 0.001$ & 1.368 & 0.825 & 0.345 & 3.96 & 2.39 \\
\hline $\mathbf{T}_{S}$ & $0.110 \pm 0.003$ & $0.121 \pm 0.002$ & $0.053 \pm 0.002$ & 0.823 & 1.003 & 0.311 & 2.64 & 3.23 \\
\hline$k^{\mathrm{a}}$ & & & & 1.66 & 1.22 & & & \\
\hline \multicolumn{3}{|c|}{ Model $/ T_{R}$} & \multicolumn{3}{|l|}{$\operatorname{MIP5}_{R}$} & \multicolumn{3}{|c|}{ NIP5 } \\
\hline \multicolumn{9}{|c|}{ Langmuir } \\
\hline \multicolumn{3}{|c|}{$\mathrm{Q}_{\max }\left(\mu \mathrm{mol} \mathrm{g}^{-1}\right)$} & \multicolumn{3}{|l|}{0.261} & \multicolumn{3}{|c|}{0.167} \\
\hline \multicolumn{3}{|c|}{ 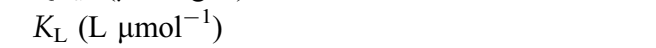 } & \multicolumn{3}{|l|}{0.471} & \multicolumn{3}{|c|}{0.370} \\
\hline \multicolumn{3}{|c|}{$R_{\mathrm{L}}\left(c=0.25,0.5,0.75,1.25,10 \mu \mathrm{mol} \mathrm{L}{ }^{-1}\right)$} & \multicolumn{3}{|c|}{$0.89 ; 0.81 ; 0.74 ; 0.63 ; 0.18$} & \multicolumn{3}{|c|}{$0.92 ; 0.84 ; 0.78 ; 0.68 ; 0.21$} \\
\hline \multicolumn{3}{|c|}{$r^{2}$} & \multicolumn{3}{|c|}{0.897} & \multicolumn{3}{|c|}{0.988} \\
\hline \multicolumn{9}{|c|}{ Dubinin-Radushkevich } \\
\hline \multicolumn{3}{|c|}{$E\left(\mathrm{~kJ} \mathrm{~mol}^{-1}\right)$} & \multicolumn{3}{|l|}{1.71} & \multicolumn{3}{|c|}{2.84} \\
\hline \multicolumn{3}{|c|}{$q_{\mathrm{s}}\left(\mu \mathrm{mol} \mathrm{g} \mathrm{g}^{-1}\right)$} & \multicolumn{2}{|l|}{1.054} & & \multicolumn{3}{|c|}{0.172} \\
\hline \multicolumn{3}{|l|}{$r^{2}$} & \multicolumn{2}{|l|}{0.975} & & \multicolumn{3}{|c|}{0.968} \\
\hline
\end{tabular}

${ }^{\text {a }}$ for $\mathrm{MIP}_{R}: k=\mathbf{T}_{\boldsymbol{R}} / \mathbf{T}_{S} ;$ for $\mathrm{MIP}_{S}: k=\mathbf{T}_{S} / \mathbf{T}_{\boldsymbol{R}}$ 
the free energy of sorption could be calculated, together with the dimensionless constant separation parameter $\left(R_{\mathrm{L}}\right)$ (eq. S6). The results are presented in Table 2. The maximum monolayer coverage capacity $\left(Q_{\max }\right)$ was higher for MIP5 $5_{R}$ than for NIP5 and also the Langmuir constant $\left(K_{\mathrm{L}}\right)$. The value of $R_{\mathrm{L}}$ indicates whether the adsorption is unfavourable $\left(R_{\mathrm{L}}>1\right)$, linear $\left(R_{\mathrm{L}}=1\right)$, favourable $\left(1>R_{\mathrm{L}}>0\right)$ or irreversible $\left(R_{\mathrm{L}}=0\right)$. Here, in the tested concentrations, the adsorption process of $T_{R}$ was found to be favourable on MIP5 $5_{R}$ and NIP5. The linear regression coefficient was good for NIP5 but moderate for $\mathrm{MIP}_{R}$.

Next, the Dubinin-Radushkevich model was employed [35]. This model was introduced to express the impact of a porous adsorbent structure and was based on the adsorption potential theory. The model is based on the assumption that the adsorption process occurs on a heterogeneous surface. For this reason, the Dubinin-Radushkevich model was considered to be more fitting than the Langmuir model. The linear form of the Dubinin-Radushkevich isotherm is presented in eq. S7. Mean free energy calculated using eq. S8 indicated whether the adsorption process was governed by physical or chemical interactions. The magnitude of mean adsorption energy $(E)$ below $8 \mathrm{~kJ} \mathrm{~mol}^{-1}$ indicated the physisorption process, but the value of $E$ above $8 \mathrm{~kJ} \mathrm{~mol}^{-1}$ indicated chemisorption. Here, the values of $E$ indicated the physisorption process of $\mathbf{T}_{R}$ on both tested materials. The linear regression coefficient was very good, confirming that the DubininRadushkevich model fitted the experimental data best. To sum up, the physisorption process of $\mathbf{T}_{R}$ on $\mathrm{MIP5}_{R}$ was confirmed by employing the DubininRadushkevich model.

\section{Theoretical insight into polymer cavities imprinted by a chiral template}

In order to provide an insight into the enantiomeric differentiation ability of MIPs, a theoretical analysis of interactions in the cavities formed by each enantiomer of the $T$ was carried out. Equation $S 4$ was used to calculate the $\Delta E_{\mathrm{B}}$ for both enantiomers, and $\mathrm{T}_{R}$ and $\mathrm{T}_{S}$ inside the polymer cavities of MIP5 $5_{R}$ and MIP5 $5_{S}$. A stronger interaction with MIP5 $5_{R}$ was predicted for $\mathrm{T}_{R}$ $\left(\Delta E_{\mathrm{B}}=-506.14 \mathrm{kcal} \mathrm{mol}^{-1}\right)$ than for $\mathrm{T}_{S}\left(\Delta E_{\mathrm{B}}=\right.$ $\left.-256.77 \mathrm{kcal} \mathrm{mol}^{-1}\right)$. The $\Delta E_{\mathrm{B}}$ values inside the $\mathrm{MIP5}_{S}$ cavity for both $\mathrm{T}$ enantiomers showed the same trend, with $\Delta E_{\mathrm{B}}$ values obtained for $\mathbf{T}_{S}$ and $\mathbf{T}_{R}$ equal to -347.18 and $-302.42 \mathrm{kcal} \mathrm{mol}^{-1}$, respectively. According to the theoretical calculations, the $R$-enantiomer matched the $R$-cavity, and the $S$-enantiomer matched the $S$-cavity. This finding is in an agreement with other experimental data [23] and could be proof that the theoretical models proposed in this paper are capable of evaluating stereo-differentiation of MIPs. Figure 4 shows the locations of $\mathbf{T}_{R}$ and $\mathbf{T}_{S}$ in the cavities of MIP5 $5_{R}$ and MIP5 $5_{S}$, together with overlaid conformations of both enantiomers (on the right side). Different steric requirements could be observed in both cavities for $\mathbf{T}_{R}$ and $\mathbf{T}_{S}$. Molecules were located in the binding pockets in such a way that they maximized favourable contacts. Orientation of $\mathrm{T}_{R}$ in the $\mathrm{MIP}_{R}$ cavity was different to the orientation of $\mathbf{T}_{S}$.

The difference was related to the location of the $-\mathrm{NH}_{2}$ group as well as to the steric location of the acyclic ring of the $T$. Amine groups of $T_{R}$ and $T_{S}$ were located opposite each other in the MIP5 $5_{R}$ cavity. In the MIP5 $5_{S}$ cavity, the $-\mathrm{NH}_{2}$ groups of both enantiomers were situated in the same direction and in a fairly similar way, but the ring part of both molecules was placed in a totally different way. Comparison of the steric locations of $\mathbf{T}_{R}$ and $\mathbf{T}_{S}$ in both cavities, together with comparison of $\Delta E_{\mathrm{B}}$ values, showed that the $-\mathrm{NH}_{2}$ group played the most important role in the molecular recognition mechanism of the systems analysed. In the $R$-cavity (where the $-\mathrm{NH}_{2}$ group of both enantiomers had a different location), the difference between $\Delta E_{\mathrm{B}}$ values for $\mathbf{T}_{R}$ and $\mathrm{T}_{S}$ was significant $\left(\Delta E_{\mathrm{B}}=-506.14\right.$ and $-256.77 \mathrm{kcal} \mathrm{mol}^{-1}$, respectively). In the $\mathrm{MIP}_{S}$ cavity (where the $-\mathrm{NH}_{2}$ group of both enantiomers was in a similar location), the difference between $\Delta E_{\mathrm{B}}$ values for $\mathbf{T}_{S}$ and $\mathrm{T}_{R}$ was less pronounced $\left(-347.18\right.$ and $-302.42 \mathrm{kcal} \mathrm{mol}^{-1}$, respectively).

To further understand the conformational preferences of $\mathbf{T}_{R}$ and $\mathbf{T}_{S}$ in the cavities of MIP5 $5_{R}$ or MIP5 $5_{S}$, two factors were analysed, viz. the distribution of atomic partial charges (ESP) in $\mathbf{T}_{R}$ and $\mathbf{T}_{S}$ molecules and the intermolecular interactions formed between $\mathbf{T}_{R}$ or $\mathbf{T}_{S}$ and polymer residues.

The distribution of atomic charges on $\mathbf{T}_{R}$ and $\mathbf{T}_{S}$ revealed distinct variations between both enantiomers, mainly in the regions of $\mathrm{N}$ and $\mathrm{C}$ atoms of the acyclic ring of both molecules (Fig. S1). These variations could be responsible for the stability of respective PCs and the distribution of monomer and 
Figure 4 The positions of $\mathbf{T}_{R}$ (brown colour) and $\mathbf{T}_{S}$ (pink colour) in the MIP5 $5_{R}$ and MIP5 $_{S}$ cavities.
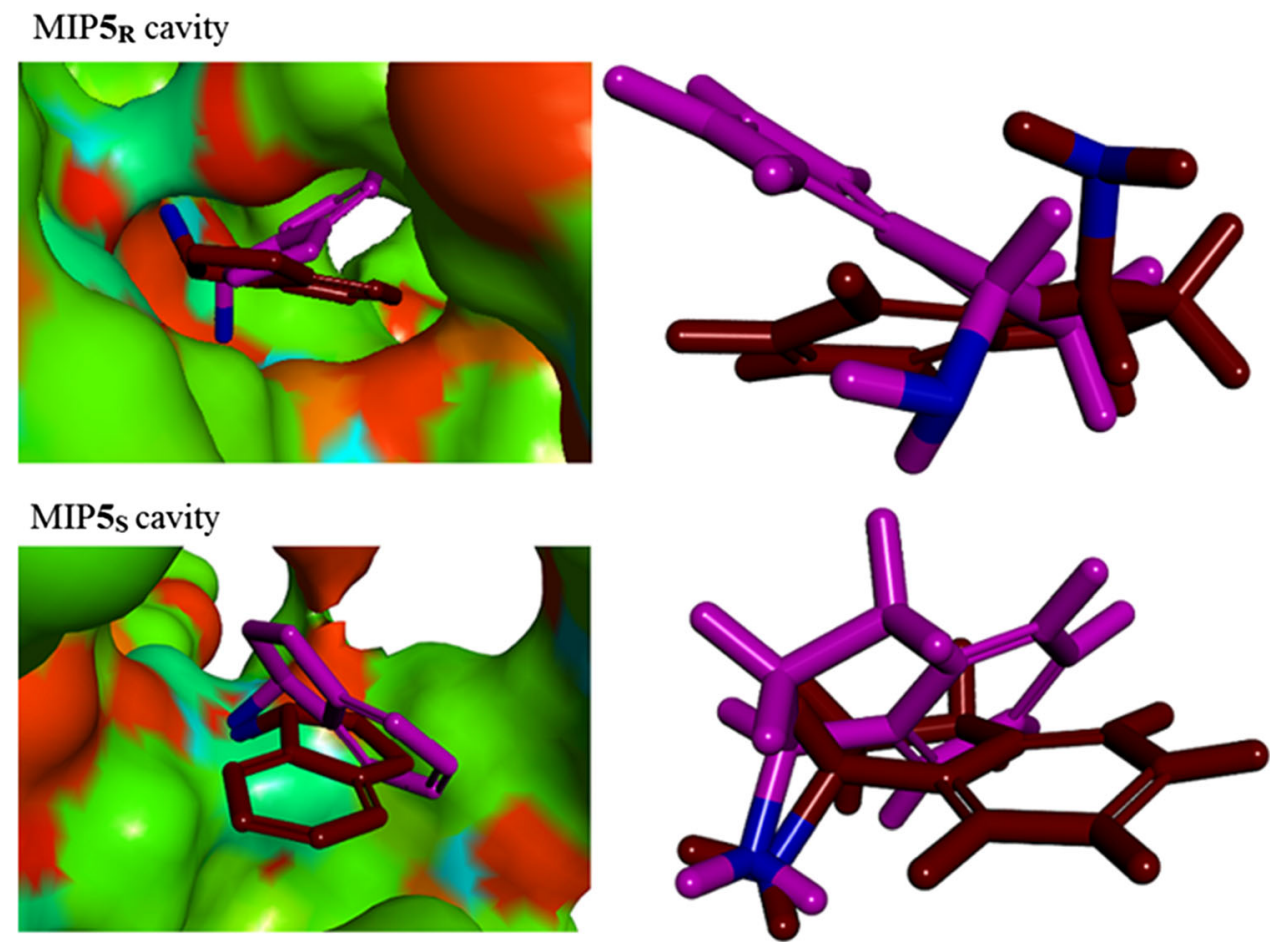

cross-linker molecules around $\mathbf{T}_{R}$ and $\mathbf{T}_{S}$ and could also be responsible for the strength of interactions between $\mathbf{T}_{R}$ or $\mathbf{T}_{S}$ and polymer residues in the cavity during the adsorption process. Thus, the intermolecular interactions formed between $T_{R}$ or $T_{S}$ and polymer residues were examined by analysing $\mathrm{MD}$ results. Figure 5 presents the strongest interactions formed between $T_{R}$ or $T_{S}$ and residues in both cavities at the end of the adsorption process.

The $T_{R}$-MIP5 $F_{R}$ system was stabilized by four hydrogen bonds and six hydrophobic interactions (Fig. 5a). Hydrogen bonds were observed between $\mathrm{O}$ or $\mathrm{H}$ atoms of the $-\mathrm{COOH}$ groups in the monomer residues and $\mathrm{H}$ or $\mathrm{N}$ atoms in the $-\mathrm{NH}_{2}$ group of $\mathrm{T}_{R}$ (ranging from 2.02 to $2.34 \AA$ ). Hydrophobic interactions were found between $-\mathrm{CH}_{3}$ groups of monomer or cross-linker residues and $\pi$-electrons or the cycloalkyl part of the indane ring of $\mathbf{T}_{R}$ (ranging from 4.30 to $5.31 \AA$ ).

The $\mathbf{T}_{S}$ enantiomer in the MIP5 $5_{R}$ cavity (Fig. $5 \mathrm{~b}$ ) only formed one weak non-classical hydrogen bond between the $\mathrm{O}$ atom of the $-\mathrm{COOH}$ group in the monomer residue and the $-\mathrm{CH}$ - group of the indane ring (substituted by the $-\mathrm{NH}_{2}$ group of $\mathrm{T}_{S} ; 2.34 \AA$ ). Additionally, the indane ring (cycloalkyl part and $\pi$ electrons) of $\mathbf{T}_{S}$ created seven hydrophobic interactions with $-\mathrm{CH}_{3}$ groups of monomer or cross-linker residues (ranging from 4.30 to $5.46 \AA$ A). Moreover, significantly lower adsorption of $\mathbf{T}_{S}$ than $\mathbf{T}_{R}$ on MIP $_{R}$ could be the result of interaction of $\mathbf{T}_{S}$ with solvent molecules where interactions between methanol molecules and $\mathbf{T}_{S}$ (non-classical hydrogen bonds between the $\mathrm{H}$ atom of the $-\mathrm{CH}_{3}$ group of methanol and $\mathrm{N}$ atom of $\mathrm{T}_{S}$ and $\pi$-lone pair interaction) were observed. Weaker interaction of $\mathbf{T}_{S}$ with $M I P 5_{R}$ could be a consequence of the presence of unfavourable steric hindrance between the $\mathrm{O}$ atom of the cross-linker and $-\mathrm{NH}_{2}$ group of $\mathbf{T}_{S}$.

A similar analysis was performed of interactions between the MIP5 $5_{S}$ cavity and both the $T_{S}$ and $T_{R}$ enantiomers (Figs. 5c, d, respectively). In the MIP $5_{S}$ cavity, the $\mathrm{H}$ atom from the $-\mathrm{COOH}$ group in the monomer residue interacted with the $\mathrm{N}$ atom of the $\mathbf{T}_{S}$, creating a hydrogen bond $(2.22 \AA)$, and the $\mathrm{O}$ atom from the $-\mathrm{COOH}$ group in the monomer residue formed a non-classical hydrogen bond with the - $\mathrm{CH}$ - group of the indane ring (substituted by the $-\mathrm{NH}_{2}$ group of $\mathrm{T}_{S} ; 2.97 \AA$ ). Furthermore, three hydrophobic interactions were present between $-\mathrm{CH}_{3}$ groups of cross-linker residues and $\pi$-electrons or the alkyl part of the indane ring of $\mathbf{T}_{S}$ (ranging from 4.42 to $5.29 \AA$ ). In the $\mathrm{MIP5}_{S}$ cavity, $\mathrm{T}_{R}$ formed the same type of hydrogen bonds between the $\mathrm{H}$ atom of the $-\mathrm{COOH}$ group in the monomer residue and the $\mathrm{N}$ 
(a)

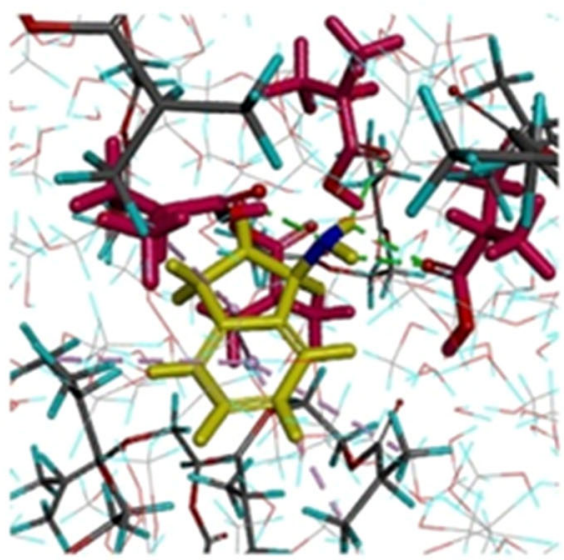

(c)

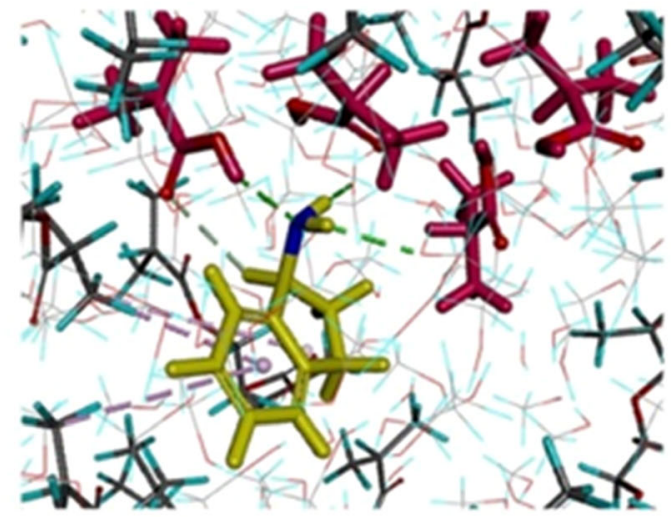

Figure 5 Views of $\mathrm{T}_{R}$ and $\mathrm{T}_{S}$ in the cavities of $\mathrm{MIP}_{R}$ and MIP5 at the end of adsorption process simulation: $\mathbf{a} \mathbf{T}_{R}$ in $\mathrm{MIP5}_{R} ; \mathbf{b} \mathbf{T}_{S}$ in $\mathrm{MIP}_{R} ; \mathrm{c} \mathrm{T}_{S}$ in $\mathrm{MIP}_{S} ; \mathrm{d} \mathrm{T}_{R}$ in MIP5 $5_{S}$. The classical hydrogen bonds are shown as the dashed green lines, non-classical hydrogen

atom of $\mathbf{T}_{R}(2.24 \AA)$ and two hydrophobic interactions with the monomer and cross-linker residues (4.54 and $5.11 \AA$ ). The lower values of $\Delta E_{\mathrm{B}}$ for the $\mathrm{T}_{S^{-}}$ $\mathrm{MIP5}_{S}$ system in comparison with the $\mathrm{T}_{R}-\mathrm{MIP}_{R}$ system $\left(-506.14\right.$ and $-347.18 \mathrm{kcal} \mathrm{mol}^{-1}$, respectively) could also be a result of different atomic charge distribution on $\mathbf{T}_{S}$.

According to the theoretical calculations, the highest impact on stereo-differentiation was due to interactions formed by the $-\mathrm{NH}_{2}$ group of $\mathrm{T}_{S}$ or $\mathrm{T}_{R}$. Hence, it could be concluded that the models of $\mathrm{MIP}_{R}$ and MIP5 $5_{S}$ could differentiate between $R$ - and $S$-enantiomers and could be used to facilitate explanation of the adsorption process of chiral molecules on MIPs.

To sum up, higher $B$ and higher chiral recognition ability were noted for $\mathrm{MIP}_{R}$ towards $\mathrm{T}_{R}$ than for $\mathrm{MIP}_{S}$ towards $\mathrm{T}_{S}$.

(b)

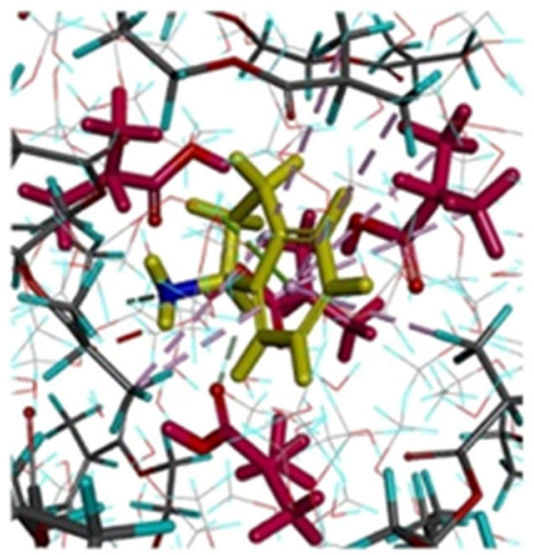

(d)

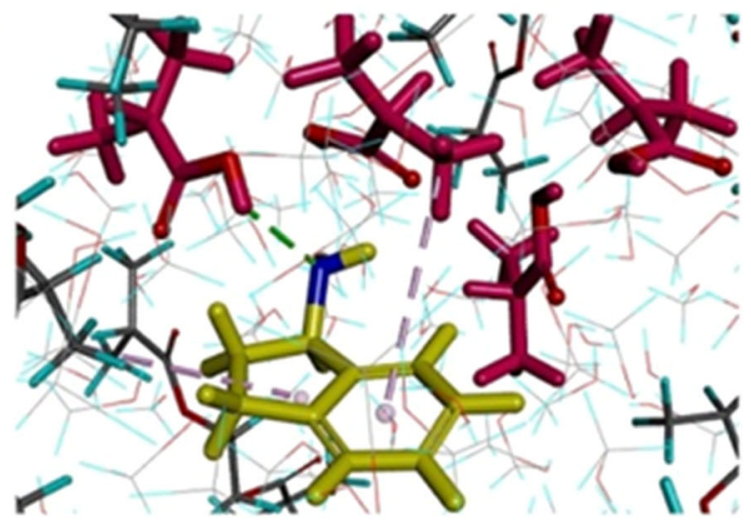

bonds are shown as the grey dashed lines, hydrophobic interactions are shown as the dashed pink lines, and unfavourable steric hindrance (in $\mathbf{b}$ ) is shown as the dashed red.

Impact of absolute template configuration on surface morphology and the pore system of the bulk imprinted polymer

Bulk imprinted material is often produced during preliminary screening of MIPs' binding abilities due to the low cost and simplicity of synthesis. Here, $\mathrm{MIP5}_{R}, \mathrm{MIP5}_{S}$ and NIP5 bulk polymers were obtained, and their surface morphology and pore systems were analysed using SEM and BET analyses.

The polymer surfaces were investigated first. The micrographs of $\mathrm{MIP5}_{R}, \mathrm{MIP}_{S}$ and NIP5 (Fig. 6), prepared in the bulk process, confirmed the typical morphology of particles (i.e. an irregular shape and size, a diameter of ca. $20-40 \mu \mathrm{m}$ and a heterogeneous surface), with areas of high extension and areas of rupture without pores (Figs. 6a-c). Higher magnification of the micrographs revealed a negligible difference between $M I P 5_{R}$ and $M I P 5_{S} . M I P 5_{R}$ and $M I P 5_{S}$ 

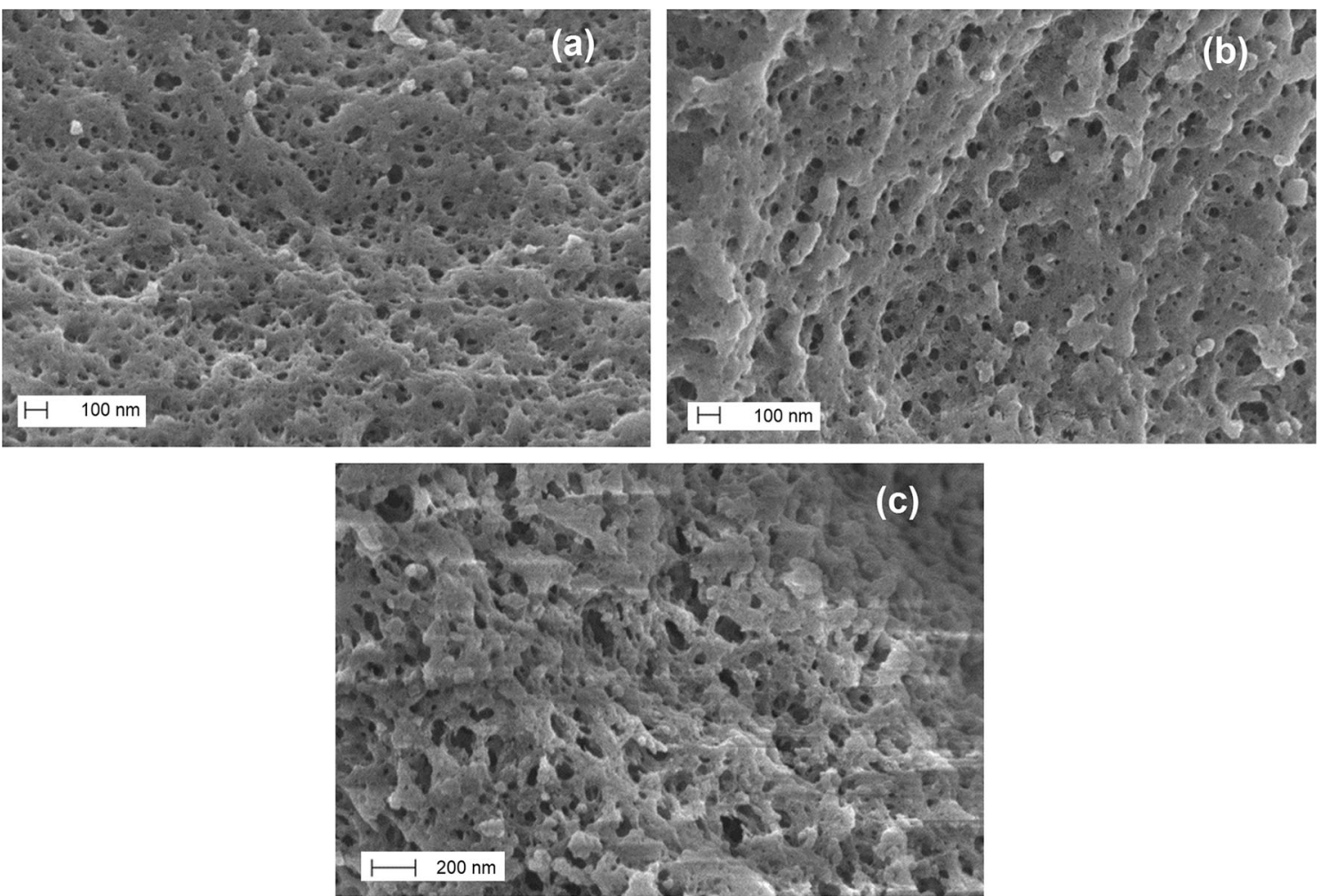

Figure 6 Micrographs of bulk polymers: $\operatorname{MIP}_{R}(\mathbf{a}), \mathrm{MIP}_{S}(\mathrm{~b})$ and NIP5 (c).

were characterized by moderate surface extension and numerous pores, $40-100 \mathrm{~nm}$ in diameter (Fig. S2a). In contrast, NIP5 possessed a highly extended, sponge-like surface with numerous pores, 30-150 nm in diameter (Fig. S2b).

Analysis of pore structure is essential, since it can explain the properties of an adsorbent, which could be an important parameter in terms of the efficiency of an imprinting process. In studies like this, 77-K nitrogen sorption analysis is the technique commonly used to provide data that fit the relevant mathematical models. Thus, in order to evaluate the impact of the composition of prepolymerization mixtures on pore structures of resultant polymers, the macroporous and microporous systems of $\mathrm{MIP}_{R}, \mathrm{MIP} 5_{S}$ and NIP5 were analysed.

Firstly, nitrogen adsorption isotherms (BrunauerEmmett-Teller isotherm) were analysed [36]. Figure 7 presents nitrogen adsorption isotherms for $\mathrm{MIP}_{R}, \mathrm{MIP}_{S}$ and NIP5.

As can be seen, all imprinted materials revealed type IV physisorption isotherms, which characterize mesoporous sorbents with unrestricted mono/multilayered adsorption [37]. The materials also all revealed hysteresis loops. Hysteresis loop shapes are

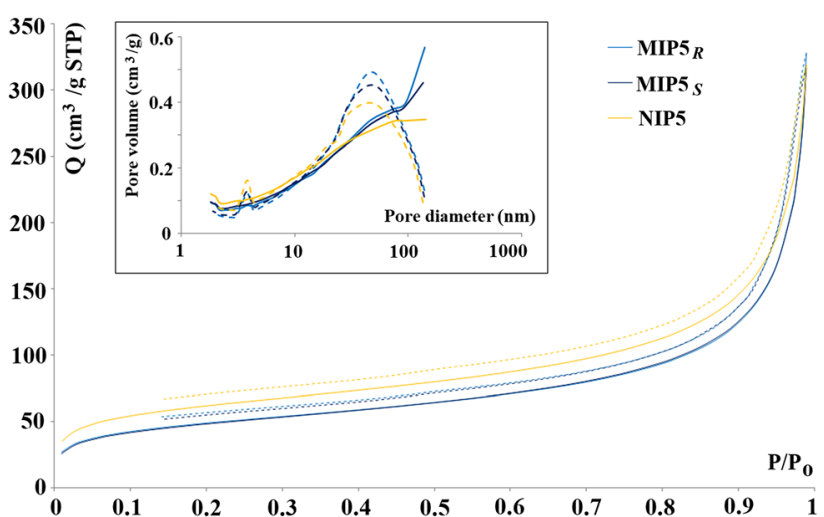

Figure 7 Nitrogen adsorption isotherms for $\mathrm{MIP}_{R}$ (light blue), MIP5 $_{S}$ (dark blue), NIP5 (orange)-adsorption lines are continuous, desorption lines are dashed $\left(P_{\mathrm{o}}\right.$ is the nitrogen saturation pressure). Inset presents pore-size distributions calculated using the Barrett-Joyner-Halenda model on both the adsorption and the desorption branches of isotherms for MIP5 $5_{R}$ (light blue), MIP5 $5_{S}$ (dark blue) NIP5 (orange)_adsorption lines are continuous, and desorption lines are dashed.

related to the specific pore structure. Here, H3-type loops characterized $\mathrm{MIP}_{R}, \mathrm{MIP}_{S}$ and NIP5, indicating a slit-shape structure of the pores of all materials. Low-pressure hystereses were observed for all 
materials, and the lack of closing points could be explained by the experimental conditions rather than porosity of the sorbents [38].

Next, the total specific surface area (BrunauerEmmett-Teller isotherm) was determined, together with the cumulative pore surface area (Barrett-Joyner-Halenda model), and the volume and area of micropores (Harkins-Jura equation) [39, 40]. A higher specific surface area was noted for NIP5 $\left(223.7 \mathrm{~m}^{2} \mathrm{~g}^{-1}\right)$, and a lower one was noted for both $\mathrm{MIP5}_{R}$ and $\mathrm{MIP5}_{S}\left(177.2\right.$ and $175.0 \mathrm{~m}^{2} \mathrm{~g}^{-1}$, respectively). These findings correlated well with the surface morphology analysis (Figs. 6a-c). MIP5 $\boldsymbol{R}_{\boldsymbol{R}}$ was found to have the highest micropore area. The micropore area and external surface area of NIP5 were also higher than those of the MIPs (60.6/ $162.8 \mathrm{~m}^{2} \mathrm{~g}^{-1}$ for NIP5; 39.7/137.5 and 34.0/ $141.0 \mathrm{~m}^{2} \mathrm{~g}^{-1}$ for $\mathrm{MIP}_{R}$ and MIP5 $5_{S}$, respectively). The highest pore volume was noted for NIP5 $\left(0.024 \mathrm{~cm}^{3} \mathrm{~g}^{-1}\right)$, but a significant difference in pore volume was noted between MIP5 $5_{R}$ and MIP5 $5_{S}(0.015$ and $0.012 \mathrm{~cm}^{3} \mathrm{~g}^{-1}$, respectively). Most notably, the total pore volume for all materials was nearly the same $\left(0.507,0.491\right.$ and $0.493 \mathrm{~cm}^{3} \mathrm{~g}^{-1}$ for $\mathrm{MIP5}_{R}$, $\mathrm{MIP5}_{S}$ and NIP5, respectively). To provide more insight into the characterization of pore structures, pore-size distributions were analysed. The inset in Fig. 7 presents pore-size distributions (calculated using the Barrett-Joyner-Halenda model) on both the adsorption and desorption branches of the isotherms for $\mathrm{MIP}_{R}, \mathrm{MIP}_{S}$ and NIP5.

Pore volume versus pore diameter plots (calculated from desorption isotherms) revealed the following peaks: $50.4,50.9$ and $54.1 \mathrm{~nm}$ for $\mathrm{MIP}_{R}, \mathrm{MIP}_{S}$ and NIP5, respectively. Slight differences in pore diameter indicated that NIP5 had a more macroporous system with respect to both imprinted polymers.

To conclude, the impact of the template on poresize distribution was revealed. However, the absolute configuration of the template did not affect pore diameter.

\section{Analysis of polymers}

In order to reveal the structural similarity of the tested polymers, time-domain terahertz spectroscopy was applied. This instrumental technique provides information about the morphology and conformation of polymers, since in the terahertz regime, the electromagnetic waves interact with collective motions of large molecules, such as inter- and intramolecular vibrations of polymer chains. It was supposed that it would be possible to detect differences between $\mathrm{MIP}_{R}, \mathrm{MIP}_{S}$ and NIP5 because of variations in their morphology and binding properties. Figure 8 presents $\mathrm{MIP}_{R}, \mathrm{MIP}_{S}$ and NIP5 spectra in the terahertz range.

As can be seen, the absorption curves for MIP5 $5_{R}$, $\mathrm{MIP}_{S}$ and NIP5 are characterized by the same trend. The adsorption coefficient increased with increasing frequency. The spectra did not possess any characteristic peaks in the range below $3 \mathrm{THz}$. Only negligible differences were observed (related to the flatter curves of MIPs in comparison with those of NIP5). The calculated refractive indices were very similar, with values equal to 1.195 for both MIP5 ${ }_{R}$ and $M I P 5_{S}$, and 1.180 for NIP5. Thus, it could be concluded that $\mathrm{MIP5}_{R}, \mathrm{MIP5}_{S}$ and NIP5 possessed a similar structure.
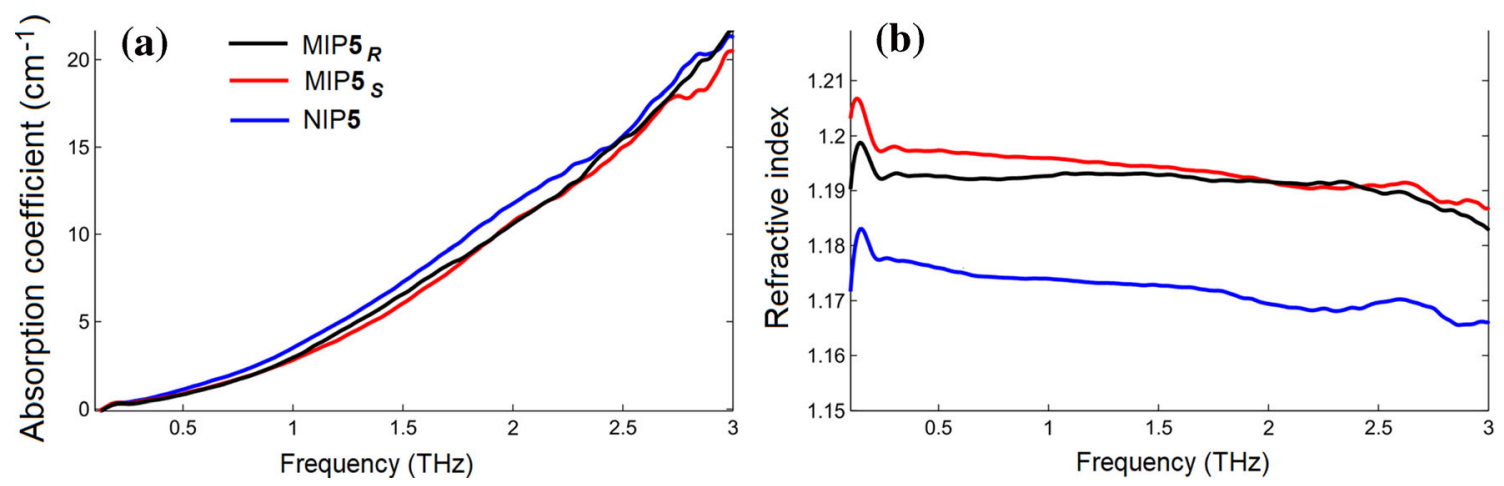

Figure 8 Absorption spectra (a) and refractive indeces (b) of MIP5 $5_{R}, M I P 5_{S}$ and NIP5 in terahertz range. 


\section{Conclusions}

Optimization of synthetic protocols allowed us to obtain imprinted material with satisfactory specificity towards 1-aminoindane. The polymer synthesized from methacrylic acid was characterized by the highest specificity, expressed as an imprinting factor equal to 3 . The scientific assumption that the molecularly imprinted polymer differentiates enantiomers because of different steric requirements in both cavities obtained for each enantiomer was confirmed by theoretical as well as empirical data on binding capacities. Different steric requirements were observed in both cavities for $\mathbf{T}_{R}$ and $\mathbf{T}_{S}$. The highest impact on stereo-differentiation derived from interactions formed by the $-\mathrm{NH}_{2}$ group of $\mathbf{T}_{S}$ or $\mathbf{T}_{R}$. The $M I P 5_{R}$ and $M I P 5_{S}$ models were found to differentiate between $R$ - and $S$-enantiomers and can be used to facilitate explanation of the adsorption process of chiral molecules on MIPs. The impact of morphology on the adsorption of respective $R$ - and $S$-enantiomers on $R$ - and $S$-imprinted polymers was negligible, but a significant difference in binding capacity was noted. The structural identity of $R$ - and $S$-imprinted polymers was confirmed, proving the feasibility of terahertz spectroscopy for analysis of imprinted materials.

\section{Acknowledgements}

The results presented in this paper were obtained using the resources of the Interdisciplinary Centre for Mathematical and Computational Modelling (ICM) at the University of Warsaw, Poland (G68-2).

\section{Compliance with ethical standards}

Conflict of interest There are no conflicts to declare.

Electronic supplementary material: The online version of this article (https://doi.org/10.1007/s108 53-020-04796-z) contains supplementary material, which is available to authorized users.

Open Access This article is licensed under a Creative Commons Attribution 4.0 International License, which permits use, sharing, adaptation, distribution and reproduction in any medium or format, as long as you give appropriate credit to the original author(s) and the source, provide a link to the
Creative Commons licence, and indicate if changes were made. The images or other third party material in this article are included in the article's Creative Commons licence, unless indicated otherwise in a credit line to the material. If material is not included in the article's Creative Commons licence and your intended use is not permitted by statutory regulation or exceeds the permitted use, you will need to obtain permission directly from the copyright holder. To view a copy of this licence, visit http://creativecom mons.org/licenses/by/4.0/.

\section{References}

[1] Bradshaw DS, Leeder JM, Coles MM, Andres DL (2015) Signatures of material and optical chirality: origins and measures. Chem Phys Lett 626:106-110

[2] Dorca Y, Greciano EE, Valera JS, Gomez R, Sanchez L (2019) Hierarchy of asymmetry in chiral supramolecular polymers: toward functional, helical supramolecular structures. Chem Eur J 25:5848-5864

[3] Boon G, van Alsenoy C, de Proft F, Bultinck P, Geerlings P (2003) Similarity and chirality: quantum chemical study of dissimilarity of enantiomers. J Phys Chem A 107:11120-11127

[4] Cheong WJ, Ali F, Choi JH, Lee JO, Sung KY (2013) Recent applications of molecular imprinted polymers for enantio-selective recognition. Talanta 106:45-59

[5] Rutkowska M, Płotka-Wasylka J, Morrison C, Wieczorek PP, Namieśnik J, Marć M (2018) Application of molecularly imprinted polymers in analytical chiral separations and analysis. Trends Anal Chem 102:91-102

[6] Luliński P, Dana M, Maciejewska D (2014) Synthesis and characterization of 4-(2-aminoethyl)aniline imprinted polymer as a highly effective sorbent of dopamine. Talanta 119:623-631

[7] Luliński P, Maciejewska D (2012) Effective separation of dopamine from bananas on 2-(3,4-dimethoxyphenyl)ethylamine imprinted polymer. J Sep Sci 35:1050-1057

[8] Luliński P, Giebułtowicz J, Wroczyński P, Maciejewska D (2015) A highly selective molecularly imprinted sorbent for extraction of 2-aminothiazoline-4-carboxylic acid-synthesis, characterization and application in post-mortem whole blood analysis. J Chromatogr A 1420:16-25

[9] Marć M, Kupka T, Wieczorek PP, Namieśnik J (2018) Computational modeling of molecularly imprinted polymers as a green approach to the development of novel analytical sorbents. Trends Anal Chem 98:64-78 
[10] Sobiech M, Bujak P, Luliński P, Proń A (2019) Semiconductor nanocrystal-polymer hybrid nanomaterials and their application in molecular imprinting. Nanoscale 11:12030-12074

[11] Iskierko Z, Sharma PS, Bartold K, Pietrzyk-Le A, Noworyta K, Kutner W (2016) Molecularly imprinted polymers for separating and sensing of macromolecular compounds and microorganisms. Biotechnol Adv 34:30-46

[12] Dąbrowski M, Lach P, Cieplak M, Kutner W (2018) Nanostructured molecularly imprinted polymers for protein chemosensing. Biosens Bioelectron 102:17-26

[13] Luliński P (2017) Molecularly imprinted polymers based drug delivery devices: a way to application in modern pharmacotherapy. A review. Mater Sci Eng, C 76:1344-1353

[14] Tuwahatu CA, Yeung CC, Lam YW, Roy VAL (2018) The molecularly imprinted polymer essentials: curation of anticancer, ophthalmic, and projected gene therapy drug delivery systems. J Control Release 287:24-34

[15] Yu X, Zeng H, Wan J, Cao X (2020) Computational design of a molecularly imprinted polymer compatible with an aqueous environment for solid phase extraction of chenodeoxycholic acid. J Chromatogr A 1609:460490

[16] Sajini T, Renjith T, Beena M (2019) Rational design and synthesis of photo-responsive molecularly imprinted polymers for the enantioselective intake and release of Lphenylalanine benzyl ester on multiwalled carbon nanotubes. Polymer 173:127-140

[17] He P, Zhu H, Ma Y, Liu N, Niu X, Wei M, Pan J (2019) Rational design and fabrication of surface molecularly imprinted polymers based on multi-boronic acid sites for selective capture glycoproteins. Chem Eng J 367:55-63

[18] Spivak DA, Campbell J (2001) Systematic study of steric and spatial contributions to molecular recognition by noncovalent imprinted polymers. Analyst 126:793-797

[19] Tiwari MP, Prasad A (2015) Molecularly imprinted polymer based enantioselective sensing devices: a review. Anal Chim Acta 853:1-18

[20] Ouyang R, Lei J, Ju H, Xue Y (2007) A Molecularly imprinted copolymer designed for enantioselective recognition of glutamic acid. Adv Funct Mater 17:3223-3230

[21] Sekine S, Watanabe Y, Yoshimi Y, Hattori K, Sakai K (2007) Influence of solvents on chiral discriminative gate effect of molecularly imprinted poly(ethylene glycol dimethacrylateco-methacrylic acid). Sens Actuators, B 127:512-517

[22] Terracina JJ, Sharfstein ST, Bergkvist M (2018) In silico characterization of enantioselective molecularly imprinted binding sites. J Mol Recognit 31:e2612

[23] Sobiech M, Żołek T, Luliński P, Maciejewska D (2016) Separation of octopamine racemate on (R, S)-2-amino-1- phenylethanol imprinted polymer-experimental and computational studies. Talanta 146:556-567

[24] Cowen T, Karim K, Piletsky S (2016) Computational approaches in the design of synthetic receptors - a review. Anal Chim Acta 936:62-74

[25] Wietzke S, Jansen C, Reuter M, Jung T, Kraft D, Chatterjee S, Fischer BM, Koch M (2011) Terahertz spectroscopy on polymers: a review of morphological studies. J Mol Struct 1006:41-51

[26] Żołek T, Luliński P, Maciejewska D (2011) A computational model for selectivity evaluation of 2-(3,4dimethoxyphenyl)ethylamine (homoveratrylamine) imprinted polymers towards biogenic compounds. Anal Chim Acta 693:121-129

[27] Luliński P, Sobiech M, Żołek T, Maciejewska D (2014) A separation of tyramine on a 2-(4-methoxyphenyl)ethylamine imprinted polymer: an answer from theoretical and experimental studies. Talanta 129:155-164

[28] Zink S, Moura FA, da Silva Autreto PA, Galvão DS, Mizaikoff B (2018) Virtually imprinted polymers (VIPs): understanding molecularly templated materials via molecular dynamics simulations. Phys Chem Chem Phys 20:13145-13152

[29] Dassault Systèmes BIOVIA (2018) Discovery studio modeling environment, release 2019. Dassault Systèmes, San Diego

[30] Luliński P, Maciejewska D (2011) Impact of functional monomers, cross-linkers and porogens on morphology and recognition properties of 2-(3,4-dimethoxyphenyl)ethylamine imprinted polymers. Mater Sci Eng, C 31:281-289

[31] Xia Y, Zhao F, Zeng B (2020) A molecularly imprinted copolymer based electrochemical sensor for the highly sensitive detection of L-tryptophan. Talanta 206:120245

[32] Golker K, Olsson GD, Nicholls IA (2017) The influence of a methyl substituent on molecularly imprinted polymer morphology and recognition-acrylic acid versus methacrylic acid. Eur Polym J 92:137-149

[33] Long Y, Philip JYN, Schillen K, Liu F, Ye L (2011) Insight into molecular imprinting in precipitation polymerization systems using solution NMR and dynamic light scattering. J Mol Recognit 24:619-630

[34] Langmuir I (1918) The adsorption of gases on plane surfaces of glass, mica and platinum. J Am Chem Soc 40:1361-1403

[35] Stoeckli F (2001) Dubinin's theory and its contribution to adsorption science. Russ Chem Bull 50:2265-2272

[36] Brunauer S, Emmett PH, Teller E (1938) Adsorption of gases in multimolecular layers. J Am Chem Soc 60:309-319

[37] Sing KSW (1982) Reporting physisorption data for gas/solid systems with special reference to the determination of surface area and porosity. Pure Appl Chem 54:2201-2218 
[38] Silvestre-Albero AM, Juarez-Galan JM, Silvestre-Albero J, Rodríguez-Reinoso F (2012) Low-pressure hysteresis in adsorption: an artifact? J Phys Chem C 116:16652-16655

[39] Barrett EP, Joyner LG, Halenda PP (1951) The determination of pore volume and area distributions in porous substances. I. Computations from nitrogen isotherms. J Am Chem Soc $73: 373-380$
[40] Jura G, Harkins WD (1946) Surfaces of solids. XIV. A unitary thermodynamic theory of the adsorption of vapors on solids and of insoluble films on liquid subphases. J Am Chem Soc 68:1941-1952

Publisher's Note Springer Nature remains neutral with regard to jurisdictional claims in published maps and institutional affiliations. 